1 Universidade Federal de Mato Grosso do Sul (UFMS) - Campo Grande (MS), Brasil.

alessandrodecarli@hotmail. com

2 Universidade Federal de Mato Grosso do Sul (UFMS) - Campo Grande (MS), Brasil.

maralisi@globo.com

3 Universidade Federal de Mato Grosso do Sul (UFMS) - Campo Grande (MS), Brasil.

albertss@hotmail.com

4 Fundação Oswaldo Cruz Mato Grosso do Sul - Campo Grande (MS), Brasil.

esc.fiocruz@saude.ms.gov.br

5 Universidade Federal de Mato Grosso do Sul (UFMS) - Campo Grande (MS), Brasil.

apbatiston@hotmail.com

\section{Visita domiciliar e cuidado domiciliar na Atenção Básica: um olhar sobre a saúde bucal}

Home visit and home care in the Primary Care: a look on oral health

\author{
Alessandro Diogo De-Carli1, Mara Lisiane de Moraes dos Santos ${ }^{2}$, Albert Schiaveto de Souza ${ }^{\mathbf{3}}$, \\ Vera Lúcia Kodjaoglanian ${ }^{4}$, Adriane Pires Batiston ${ }^{5}$
}

RESUMO O objetivo do presente trabalho foi analisar o processo de atenção domiciliar nas Equipes de Atenção Básica que aderiram ao Programa Nacional de Melhoria do Acesso e da Qualidade. Realizou-se um estudo a partir do banco de dados do programa relativos à dimensão que avaliou a realização de visita domiciliar e cuidado domiciliar, com ênfase nas práticas das Equipes de Saúde Bucal. Quase 100\% das Equipes de Saúde da Família avaliadas realizam visita domiciliar. O cuidado no domicílio é realizado por mais de $90 \%$ dos profissionais da equipe mínima, e por aproximadamente $50 \%$ dos profissionais da saúde bucal. A saúde bucal tem desafios a superar em direção a novas práticas na Atenção Básica.

PALAVRAS-CHAVE Visita domiciliar; Saúde bucal; Atenção Primária à Saúde.

ABSTRACT The objective of the present work was to analyze the process of home care within the primary care teams that joined the National Program for Access and Quality Improvement. A study was conducted from the database of the program related to the dimension that has evaluated the accomplishment of home visit and home care, with emphasis on the practices of the oral health team. Almost 100\% of the evaluated family health teams perform home visits. The home care is performed by more than $90 \%$ of the professionals of the minimum team, and by approximately $50 \%$ of professionals of the oral health team. The oral health team has challenges to overcome towards the new practices in primary care.

KEYWORDS Home visit; Oral health; Primary Health Care. 


\section{Introdução}

O Sistema Único de Saúde (SUS), possivelmente o maior patrimônio de política pública do País, foi idealizado e constituído por um movimento da sociedade brasileira. Adota os princípios da Atenção Primária à Saúde (APS), e coloca a Estratégia Saúde da Família (ESF) como ordenadora do cuidado nas redes de atenção à saúde, com papel central para a reorganização do modelo assistencial. Contudo, os avanços políticos no setor saúde e a implantação de um número expressivo de Unidades Básicas de Saúde (UBS) não garantem a transformação deste. Esta mudança demanda modificações dos processos de trabalho, de gestão, de formação, de participação, bem como conhecimento da realidade das equipes para planejamento e viabilização de mudanças concretas no cotidiano da gestão e dos serviços, produzindo potência para a consolidação do SUS.

Mediante este contexto, a qualidade da gestão e das práticas de atenção à saúde na Atenção Básica (AB) tem sido prioridade na agenda dos gestores do SUS, e o Ministério da Saúde tem intensificado os esforços em direção a iniciativas para a qualificação da AB. Nessa conjuntura foi criado o Programa Nacional de Melhoria do Acesso e da Qualidade (PMAQ), com o principal objetivo de induzir a ampliação do acesso e a melhoria da qualidade da $\mathrm{AB}$, por meio de um processo bastante complexo que envolve quatro fases distintas: Adesão e Contratualização; Desenvolvimento; Avaliação Externa e Recontratualização. Particularmente, duas das fases configuram momentos avaliativos: o momento da Autoavaliação (fase do Desenvolvimento) e o momento da Avaliação Externa. Na fase da Avaliação Externa é realizado um conjunto de ações visando investigar as condições de acesso e de qualidade da atenção na totalidade de municípios e Equipes de Atenção Básica (EqAB) que aderiram ao programa (PINTO; SOUSA; FLORENCIO, 2013), visto que os modelos assistenciais são delineados e projetados nos microespaços e no cotidiano dos serviços por uma diversidade de aspectos inerentes aos processos de trabalho e aos serviços de saúde, situados em uma conjuntura social-econômica-cultural-histórica (REIS ET AL., 2007).

Entre os indicadores investigados por meio da Avaliação Externa do PMAQ estão a visita domiciliar e os cuidados realizados no domicílio. A primeira é destacada como um indicador de mudança do modelo de atenção (BRASIL, 2012B), alinhada aos princípios da AB, como a integralidade e a continuidade do cuidado, o acesso, a equidade, a humanização e o vínculo (BRASIL, 2012A).

A Política Nacional de Atenção Básica (BRASIL, 2012A) estabelece como uma das responsabilidades das equipes a atenção domiciliar destinada a indivíduos e famílias que tenham dificuldades para acessar os serviços. Esta é considerada como uma estratégia promotora de acesso às políticas públicas, através da relação que se estabelece entre os diferentes sujeitos do processo, como um dos instrumentos fundamentais para a atenção integral (BRASIL, 2012B).

Entretanto, ao serem mencionadas na Política Nacional de Atenção Básica as atribuições dos profissionais das equipes (BRASIL, 2012A), a visita domiciliar não está explicitada entre as atribuições do cirurgião-dentista e nem do auxiliar de saúde bucal. Por outro lado, a Política Nacional de Saúde Bucal (BRASIL, 2004), indica que as visitas domiciliares devem ser realizadas pelos profissionais da Equipe de Saúde Bucal (EqSB), como forma de ampliar o acesso aos usuários e às famílias que não têm condições de deslocamento até as unidades de saúde.

Embora se configurem como práticas essenciais na $A B$, ainda são limitados os estudos que buscam a identificação e a caracterização das práticas da visita domiciliar e os cuidados no domicílio, no cotidiano dos serviços das Unidades Básicas de Saúde Tradicionais e das Unidades Básicas de Saúde da Família no País, em especial em relação a tais práticas realizadas pelas EqSB. A literatura tem evidenciado com preocupação que, de maneira geral, os 
profissionais destas equipes reproduzem o modelo assistencial curativo, clínico, individualizado, pautado nas tecnologias duras, encontrando entraves para a mudança do modelo de atenção com a incorporação de práticas de promoção, prevenção (MATTOS ET AL., 2014) e realização pouco frequente da visita domiciliar (AQUILANTE; SILVA, 2014; FACCIN; SEBOLD; CARCERERI, 2010).

Para contribuir com o debate, a apreciação das práticas dos profissionais das EqSB em relação à visita domiciliar e ao cuidado domiciliar certamente adquire relevância nacional para a formulação de políticas indutoras de mudanças no modelo de atenção. Fato este que promove a ampliação do acesso e da qualidade da atenção à saúde de forma universal, integral e com equidade.

A análise dos dados referentes à visita domiciliar e ao cuidado no domicílio, obtidos por meio da Avaliação Externa do PMAQ junto a aproximadamente 17 mil equipes que atuam em UBS em todas as regiões do Brasil, é inédita e elucidará potencialidades e lacunas alusivas a esse tema, presentes no dia a dia das equipes. Adicionalmente, tal análise proporcionará dados concretos para o planejamento e a implementação de ações alinhadas às demandas mais evidentes identificadas na realidade dos serviços, e que serão norteadores de políticas de saúde na perspectiva da atenção integral e da ampliação do acesso à saúde bucal.

Os objetivos deste artigo são de apresentar os dados referentes à realização da visita domiciliar e aos cuidados no domicílio obtidos por meio da Avaliação Externa do primeiro ciclo do PMAQ, com ênfase na análise das práticas das EqSB; e, verificar se há diferenças entre estas práticas realizadas pelos distintos profissionais das equipes de ESF e das UBS tradicionais.

\section{Método}

Trata-se de um estudo observacional transversal em que foram analisados dados provenientes da etapa da Avaliação Externa do primeiro ciclo do $\mathrm{PMAQ}$, referentes às práticas dos profissionais das $\mathrm{EqAB}$, com ênfase nas EqSB. A fase de Avaliação Externa do PMAQ foi conduzida por instituições de ensino e pesquisa do País, na modalidade multicêntrica, e coordenada pelo Ministério da Saúde; realizada entre os anos de 2012 e 2013. Houve adesão de 17.202 equipes de saúde, todas participantes da Avaliação Externa. Esta foi realizada in loco por avaliadores previamente selecionados e qualificados para a aplicação do instrumento. Posteriormente à coleta, os dados foram enviados ao Ministério da Saúde, validados e disponibilizados às instituições parceiras.

O instrumento foi composto por quatro módulos: Módulo I - Observação na Unidade Básica de Saúde; Módulo II - Entrevista com o profissional da equipe e verificação de documentos; Módulo III - Entrevista com o usuário na Unidade Básica de Saúde; Módulo Eletrônico - Respondido pelos gestores, contendo informações complementares aos módulos anteriores.

Os sujeitos que responderam à entrevista no ato da Avaliação Externa poderiam ser profissionais enfermeiros, médicos ou profissionais de nível superior que agregassem o maior conhecimento sobre o processo de trabalho da equipe avaliada, e por ela indicados.

Os dados utilizados nesse estudo foram provenientes do Módulo II, bloco 32 (Visita domiciliar e Cuidado Realizado no Domicílio), sendo analisadas duas questões: II.32.1 "A equipe realiza a visita domiciliar?" e II.32.7 "Quais profissionais da equipe realizam cuidado domiciliar?” (BRASIL, 2012C, P. 29).

\section{Análise dos dados}

A avaliação da associação entre o tipo de equipe de saúde (UBS ou ESF) e a realização de visita e cuidado realizados no domicílio pelos cirurgiões-dentistas foi realizada por meio do teste qui-quadrado. Os resultados das demais variáveis avaliadas neste estudo foram apresentados na forma de estatística 
descritiva. A análise estatística foi realizada através do software SPSS, versão 20.0, considerando um nível de significância de $5 \%$.

Este estudo foi aprovado pelo Comitê de Ética em Pesquisa da Universidade Federal do Rio Grande do Sul, sob o protocolo $\mathrm{n}^{\circ}$ 21904.

\section{Resultados}

No primeiro ciclo do PMAQ foram avaliadas 17.202 equipes de saúde, sendo que destas, 99,6\% (17.034) referiram que realizam visita domiciliar em seu cotidiano de trabalho.

Do total de equipes avaliadas, $70,2 \%$ ( $n=12075)$ eram Equipes de Saúde da Família (EqSF) com EqSB; 26,6\% ( $n=4568)$ eram EqSF sem EqSB; 1,9\% (n=328) delas eram
EqAB (parametrizada - UBS) com EqSB; $0,7 \%(n=124)$ eram $A B$ (parametrizada UBS) sem EqSB; e 0,5\% ( $n=90)$ foram informadas como outros tipos de equipes. Além disso, não havia informação quanto ao tipo de equipe para $0,1 \%$ desta amostra $(n=17)$.

Das 12.075 EqSF com EqSB, somente 12.036 delas responderam se o cirurgião-dentista realizava ou não cuidado domiciliar, enquanto das $328 \mathrm{EqAB}$ (parametrizada - UBS) com EqSB, 319 responderam a este questionamento.

Os resultados referentes à realização de cuidado domiciliar pelos enfermeiros, médicos, técnicos/auxiliares de enfermagem, cirurgiões-dentistas e técnicos/auxiliares de saúde bucal, tanto das EqSF quanto das EqAB (parametrizada - UBS) do Brasil estão apresentados na tabela 1.

Tabela 1. Resultados referentes à realização de visitas domiciliares pelos profissionais da Atenção Básica, tanto na ESF quanto nas UBS no Brasil

\begin{tabular}{|c|c|c|c|}
\hline \multirow{2}{*}{ Variável/Região do Brasil } & \multicolumn{2}{|c|}{ Tipo de equipe } & \multirow{2}{*}{ Total } \\
\hline & ESF & UBS & \\
\hline \multicolumn{4}{|c|}{ Profissionais da equipe que realizam cuidado domiciliar: } \\
\hline \multicolumn{4}{|l|}{ Médico } \\
\hline Total $(p=0,051)$ & $92,5(15352)$ & $90,0(398)$ & $92,5(15750)$ \\
\hline \multicolumn{4}{|l|}{ Enfermeiro } \\
\hline Total $(p=0,389)$ & $99,0(16434)$ & $98,6(436)$ & $99,0(16870)$ \\
\hline \multicolumn{4}{|c|}{ Técnico/auxiliar de enfermagem } \\
\hline Total $(p=0,993)$ & $94,8(15727)$ & $94,8(419)$ & $94,8(16146)$ \\
\hline \multicolumn{4}{|l|}{ Cirurgião-dentista } \\
\hline Total $(p=0,019)$ & $56,1(6755)$ & $49,5(158)$ & $56,0(6913)$ \\
\hline \multicolumn{4}{|c|}{ Técnico/auxiliar de saúde bucal } \\
\hline Total $(p=0,001)$ & $33,8(4068)$ & $26,2(84)$ & $33,6(4151)$ \\
\hline
\end{tabular}

Não houve diferença significativa entre os enfermeiros, médicos e técnicos/auxiliares de enfermagem das EqSF e aqueles das EqAB (parametrizada - UBS), em relação à realização do cuidado domiciliar (valor de p variando entre 0,051 e 0,993). Por outro lado, os cirurgiões-dentistas e técnicos/auxiliares de saúde bucal das EqSF realizavam mais visitas domiciliares do que aqueles da $\mathrm{AB}$ (parametrizada - UBS), com p=0,019 e 0,001, respectivamente. 


\section{Discussão}

A saúde é um direito de todos, e há que se destacar e reconhecer que a saúde bucal é parte importante do processo saúde-doença de todos os brasileiros. Especificamente nesta pesquisa, a análise das práticas de mais de 12 mil EqSB, de todas as regiões do Brasil, em relação à visita domiciliar e aos cuidados no domicílio é inédita, e coloca o conhecimento da realidade como estratégia para avanços na qualidade da atenção, mudança do modelo assistencial e da consolidação do SUS.

Os dados coletados junto às EqSF e das EqAB (parametrizada - UBS) demonstram que quase a sua totalidade informa que a visita domiciliar faz parte das práticas no cotidiano dos serviços. Quando avaliados os dados referentes às EqSB, os achados mostram que aproximadamente metade dos profissionais da saúde bucal não realiza cuidado domiciliar, com resultados bem inferiores em relação aos demais profissionais pesquisados (enfermeiros, médicos e técnicos/auxiliares de enfermagem). A realização pouco frequente do cuidado domiciliar pelos cirurgiões-dentistas foi evidenciada em estudos prévios realizados em distintas realidades e em menor abrangência territorial. Os profissionais referem que não realizam a visita domiciliar por não identificarem possibilidades de atuação no espaço do domicílio (FACCIN; SEBOLD; CARCERERI, 2010; COSTA; ARAÚJO, 2013; MATTOS ET AL., 2014).

A realização do cuidado domiciliar por enfermeiros, médicos e técnicos/auxiliares de enfermagem, proporcionalmente bem superiores em relação ao realizado pelos cirurgiões-dentistas e técnicos/auxiliares de saúde bucal, possivelmente tenha ocorrido por uma somatória de fatores, como a inserção tardia da EqSB na ESF e à concepção de dependência estrutural de tecnologias duras (AQUILANTE; SILVA, 2014), associados ao paradigma biomédico ainda predominante nos processo de formação em odontologia e à dificuldade de trabalhar em equipe e com ações coletivas (FACCIN; SEBOLD; CARCERERI, 2010; MATTOS ET AL., 2014).

Enquanto os enfermeiros, médicos e técnicos/auxiliares de enfermagem foram inseridos nas EqAB na década de 1990, somente em 2004 foi lançada a Política Nacional de Saúde Bucal, que propõe a reorientação do modelo assistencial, a ampliação do acesso à saúde bucal e o cuidado em todos os níveis de atenção (BRASIL, 2004). Entretanto, ainda hoje se observa que as ações restringem-se ao atendimento clínico ambulatorial básico e individualizado, por parte do cirurgião-dentista, e a atividades tradicionais, por parte dos auxiliares em saúde bucal (instrumentação, desinfecção e esterilização de materiais /instrumentais), sem a realização prioritária de ações coletivas, como as de promoção, prevenção da saúde, visita domiciliar e atividades junto aos equipamentos sociais do território (AQUILANTE; SILVA, 2014).

$O$ trabalho em equipe e a realização de ações coletivas ainda são considerados desafios para todos os profissionais da saúde, os quais ainda têm, em sua formação, a centralidade na realização de procedimentos técnicos, individualizados e atrelados a tecnologias duras. No caso da formação dos odontólogos, essa realidade não é diferente. $\mathrm{O}$ trabalho em equipe é um importante entrave, e os processos formadores nas universidades ainda respaldam o modelo de atenção biomédico individualizado, com a utilização de equipamentos e tecnologias avançadas (MATTOS ET AL., 2014), sob forte interferência da indústria de equipamentos e insumos odontológicos e pela concepção arraigada no imaginário social da atuação profissional em consultório privado, distante das práticas compartilhadas com profissionais de outras áreas (AQUILANTE; SILVA, 2014).

A mudança paradigmática não ocorre espontaneamente, é necessário que a formação dos profissionais da saúde esteja alinhada ao sistema de saúde vigente no País. Além disso, a Educação Permanente passa a ser fundamental no processo de mudança do modelo 
assistencial, trazendo para o cotidiano dos trabalhadores a reflexão sobre suas práticas, sendo constitutiva da qualificação da atenção e da ressignificação dos fazeres dos profissionais da saúde no cuidado humano, em todos os níveis de atenção (BRASIL, 2012A).

Além destes fatores, conforme já mencionado, na atual Política Nacional da Atenção Básica (BRASIL, 2012A), a visita domiciliar não está claramente especificada como uma das atribuições do cirurgião-dentista, como consta nas atribuições dos profissionais enfermeiros, médicos e técnicos/auxiliares de enfermagem. Tal fator certamente interfere na implicação daqueles profissionais com o cuidado domiciliar. Por outro lado, a visita domiciliar está indicada claramente na Política Nacional de Saúde Bucal:

A ampliação e qualificação das ações de saúde bucal também se fazem através de organização de visitas da equipe de saúde bucal às pessoas acamadas ou com dificuldades de locomoção, visando à identificação dos riscos e propiciando o acompanhamento e tratamento necessário. (BRASIL, 2004, P. 15).

No contexto das pessoas acamadas, por meio do censo demográfico de 2010 , foi demonstrado que $23,9 \%$ da população brasileira apresentam algum grau de deficiência, sendo 3,6 milhões de pessoas com grande dificuldade de locomoção (IBGE, 2010). Tal fato nos faz refletir sobre a necessidade de que todos os profissionais da $\mathrm{AB}$ empreguem as ferramentas da visita domiciliar e do cuidado domiciliar em seu cotidiano do trabalho, contribuindo para que o direito constitucional de acesso universal, equânime e integral à saúde das pessoas que não têm condições de se deslocarem até as unidades de saúde seja implementado.

Os resultados encontrados são preocupantes, pois contrariam os pressupostos que nortearam a inclusão das EqSB na $\mathrm{AB}$, com a proposta de romper com o modelo historicamente baseado no atendimento odontológico clínico individual, excludente, tecnicista, biologicista (MATTOS ET AL., 2014), incluindo no processo de trabalho do cirurgião-dentista ações de promoção, prevenção e controle do processo saúde-doença bucal, com responsabilização pela atenção à saúde de usuários e famílias, com base no princípio da territorialização, em diferentes espaços do território e em equipe.

Tendo em vista que o domicílio faz parte do território, que muitos usuários não têm condições de deslocamento até as unidades de saúde e que é responsabilidade das EqAB atender os cidadãos conforme suas necessidades, a atenção domiciliar passou a ser atividade inerente ao processo de trabalho das EqAB (BRASIL, 2004, 2012B). A identificação de um expressivo número de EqSB que não realiza visita domiciliar e cuidados domiciliares, evidenciado por meio da Avaliação Externa do PMAQ, gera inquietação, pois indica que as especificidades do cuidado na $\mathrm{AB}$ - como equidade do acesso, vínculo, integralidade e longitudinalidade - não estão sobrevindo como prática comum no cotidiano destas equipes.

Considerando a visita domiciliar uma ação articulada entre as EqSF e as EqSB, observam-se indicativos de que ainda está presente na atuação dos cirurgiões-dentistas da $A B$ o perfil da prática laborativa tradicional, individualista e isolada (FACCIN; SEBOLD; CARCERERI, 2010), com fortes indícios de que tais profissionais permanecem presos ao biologicismo e ao ato odontológico terapêutico atrelado ao tratamento de doenças bucais com a valorização das tecnologias duras (AQUILANTE; SILVA, 2014). No que se refere a ações articuladas entre as EqSB e as EqSF, Mattos et al. (2014) identificaram que apenas $30 \%$ dos odontólogos e auxiliares de saúde bucal de 14 municípios de Minas Gerais realizavam, com frequência, ações conjuntas com as EqSF.

Em contraponto às práticas vigentes dos profissionais das EqSB, a visita domiciliar é momento profícuo para o privilégio das tecnologias leves (MERHY, 2007), qualificando 
a atenção à saúde da população - inclusive em seu caráter subjetivo - por meio da qual ocorrerá o encontro entre os atores envolvidos no trabalho vivo em ato (MERHY, 2004). No domicílio, novas configurações do cuidado em saúde se estabelecem, incorporando tecnologias leves no fazer saúde, indispensáveis para a superação do modelo de atenção pautada no paradigma biomédiCO (SILVA ET AL., 2010).

Quando a visita domiciliar não é vivenciada frequentemente no cotidiano do trabalho na $\mathrm{AB}$, o vínculo com a população adscrita fica prejudicado, o que retroalimenta o ciclo do processo de trabalho alienante e isolado dos profissionais da saúde bucal. As visitas domiciliares, sendo inseridas na prática destes profissionais como ações mais frequentes, fomentariam a aproximação com os sujeitos e com a realidade do usuário/comunidade, bem como otimizariam a força do encontro trabalhador-cuidador-usuário, constituindo-se como uma potência para a transformação das práticas de saúde (SILVA ET AL., 2010).

Cunha e Sá (2013) ressaltaram que, mesmo quando as visitas domiciliares ocorrem, não raramente ficam limitadas ao levantamento do perfil epidemiológico em saúde bucal, como base para priorização de ações e agendamento das primeiras consultas. No entanto, deveriam ser utilizadas como um dispositivo robusto para o conhecimento do contexto familiar e dos determinantes sociais que interferem no processo saúde-doença, bem como para a realização de atividades educativas ou até curativas (desde que haja infraestrutura adequada) em impossibilitados de se deslocarem à unidade de saúde (FACCIN; SEBOLD; CARCERERI, 2010).

Para que isso ocorra, é necessário que o cirurgião-dentista se reconheça como um profissional de saúde que toma o lugar do 'dentista curador' (FACCIN; SEBOLD; CARCERERI, 2010, P. 1648), atento aos problemas bucais e também ao contexto em que os mesmos têm sua origem, de forma a incorporar, no campo de suas práticas, o cuidado integral e ampliado em saúde, o que não é contemplado (salvo exceções) pela sua formação acadêmica (MATTOS ET AL., 2014).

Em relação à atuação dos recursos humanos auxiliares em saúde bucal (que são categorizados em Auxiliar de Saúde Bucal ASB e Técnico de Saúde Bucal - TSB), esta ainda é controversa e motivo de polêmicas relativas ao campo de atuação e responsabilização legal, embora seja reconhecida como necessária para a reorientação das práticas em saúde bucal, principalmente após a inserção das EqSB na ESF (ESPOSTI ET AL., 2012). Observa-se a crescente atribuição de responsabilização (às vezes, precarizada) a estes profissionais no cotidiano dos serviços (MATTOS ET AL., 2014), muito mais relacionados às atividades clínicas nas unidades do que às realizadas em outros ambientes (COSTA; ARAÚJO, 2013). Por outro lado, na literatura há relato de exceções nas quais essa relação se inverte, observando-se que auxiliares em saúde bucal realizaram visitas domiciliares em uma frequência superior a $70 \%$ em relação às ações clínicas (SANGLARD-OLIVEIRA ET AL., 2013).

$O$ processo de trabalho das EqSB na $\mathrm{AB}$ deve ser continuamente avaliado, considerando-se que estas foram incluídas na ESF em um passado relativamente recente. Isso implica um momento de transição no que se refere à formação profissional em nível de graduação, à adequação dos recursos humanos pré-existentes e do reconhecimento dos princípios da APS como norteadores de suas ações.

Há que se considerar também a historicidade da inclusão da saúde bucal nesse contexto. Embora a motivação para a mesma tenha sido relacionada à possibilidade de mudança do modelo técnico-assistencial, sabe-se que muitas das EqSB podem ter sido incorporadas à ESF devido ao incentivo financeiro destinado à gestão, que o considera essencial para o custeio do serviço odontológico (MATTOS ET AL., 2014). Tal fato pode contribuir para a permanência de práticas 
reducionistas na esfera da saúde da família, privilegiando as ações curativas e negligenciando o cuidado integral, que deve ser centrado no sujeito e na clínica ampliada.

Outros aspectos a serem apreciados são a excessiva demanda reprimida para a saúde bucal e a incapacidade dos serviços de atenção secundária de a incorporarem em suas agendas, o que acaba por sobrecarregar as EqSB. Com isso, as ações inerentes a estas, no âmbito da $\mathrm{AB}$, ficam comprometidas, e a atenção integral e a continuidade do cuidado deixam de ser priorizadas (PEREIRA ET AL., 2012), perpetuando as práticas de atenção individualizadas no âmbito dos consultórios.

Embora esses resultados sejam preocupantes e, no que se refere ao processo de trabalho, cruciais para que o acesso ao serviço de saúde qualificado seja contemplado, cabe lembrar que de forma alguma devem servir para culpabilizações/ações punitivas, pois isto contraria a orientação indutiva, agregadora e dialógica da proposta do PMAQ. Pelo contrário, tais constatações devem ser norteadoras da priorização da superação de problemas em busca da qualidade da atenção, cujo movimento deve ser conjunto (gestãoprofissionais-usuários), dialógico, embasado no fomento à cultura da negociação, com vistas às necessidades de saúde dos usuários (PINTO; SOUSA; FLORÊNCIO, 2012).

Finalmente, mediante a análise dos dados identificamos que as visitas domiciliares e o cuidado em domicílio são mais realizados pelas equipes da ESF do que pelas EqAB tradicionais (parametrizada - UBS). O mesmo ocorre quando analisados os dados referentes ao cuidado no domicílio realizado pela EqSB.

Há fortes evidências na literatura de que a ESF interferiu positivamente em alguns indicadores de saúde, como a mortalidade infantil (AQUINO ET AL., 2009) e nas internações hospitalares por causas sensíveis à $\mathrm{AB}$ (VELOSO ET AL., 2009). Entretanto, estudos referentes aos impactos da ESF sobre indicadores de saúde bucal são limitados, e os resultados positivos ainda não são evidentes (PEREIRA ET AL., 2012). Os impactos positivos identificados na literatura são referentes ao aumento da cobertura e ampliação do acesso aos serviços de atenção à saúde bucal com a implantação das EqSB na ESF (MATTOS ET AL., 2014; PEREIRA ET AL., 2012).

A presente análise dos dados da Avaliação Externa do PMAQ vem corroborar com a concepção de que a ESF tem produzido resultados profícuos em relação ao cuidado domiciliar no que se refere à saúde bucal, embora a frequência com que estes ocorram ainda necessite ser potencializada.

\section{Conclusões}

As práticas referentes à visita domiciliar e ao cuidado no domicílio das equipes analisadas demonstram que quase a totalidade dessas equipes se refere à realização de visitas domiciliares, e que o cuidado domiciliar é realizado por um percentual expressivo dos profissionais médicos, enfermeiros e técnicos/auxiliares de enfermagem. Os dados evidenciam que a realização da visita domiciliar e o cuidado em domicílio ainda não foram amplamente incorporados nos fazeres da EqSB, e que estas, vinculadas às equipes de ESF, apresentam melhores desempenhos em relação às Unidades Básicas de Saúde Tradicionais.

Constata-se que há importantes desafios a serem superados para a efetiva reorientação do modelo assistencial da AB no País, e que as EqSB ainda têm dificuldades em incorporar ações diferentes das historicamente instituídas em suas práticas. A Avaliação Externa do PMAQ foi fundamental para desvelar essa realidade, e trazer à tona a necessidade de políticas indutoras ainda mais diretivas em relação à inclusão de ações coletivas e no ambiente domiciliar da população adscrita sob a responsabilidade das EqSB, visando à transformação das práticas de atenção à saúde bucal na AB. Recomenda-se, ainda, uma análise dos dados do PMAQ que considerem possíveis 
diferenças entre as equipes avaliadas nos distintos estados e regiões do País, possibilitando intervenções direcionadas a cada contexto, considerando-se a dimensão continental do Brasil e as muitas realidades presentes nos diferentes cenários.

\section{Referências}

AQUILANTE, A. G.; SILVA, G. G. A. O cuidado em saúde bucal após a Política Nacional de Saúde Bucal "Brasil Sorridente": um estudo de caso. Ciência \& Saúde Coletiva, Rio de Janeiro. Disponível em: $<$ http://www. cienciaesaudecoletiva.com.br/artigos/artigo_int.php?id artigo $=14964>$. Acesso em: 10 abr. 2014. No prelo.

AQUINO, R.; OLIVEIRA, N. F.; BARRETO, M. L. Impact of the family health program on infant mortality in Brazilian municipalities. Am J Public Health, v. 99, n. 1, p. 87-93, jan. 2009.

BRASIL. Ministério da Saúde. Secretaria de Atenção à Saúde. Departamento de Atenção Básica. Política Nacional de Atenção Básica / Ministério da Saúde. Secretaria de Atenção à Saúde. Brasília, DF: Ministério da Saúde, 2012a. 110 p.

Ministério da Saúde. Caderno de atenção domiciliar. Brasília, DF: Ministério da Saúde, 2012b.

. Ministério da Saúde. Secretaria de Atenção à Saúde. Departamento de Atenção Básica. Documento Síntese para Avaliação Externa do Programa Nacional de Melhoria do Acesso e da Qualidade da Atenção Básica (PMAQ). Brasília, DF: Ministério de Sáude, 2012c.

Ministério da Saúde. Secretaria de Atenção à Saúde. Departamento de Atenção Básica. Coordenação Nacional de Saúde Bucal. Diretrizes da Política Nacional de Saúde Bucal. Brasília, DF: Ministério da Saúde, 2004.

COSTA, S. M.; ARAÚJO, F. F. Dental auxiliares versus community health workers: similarities and contrasts. Revista de Odontologia da UNESP, Araraquara, v. 42, n. 5, p. 350-356, set./out. 2013.
O PMAQ, em sua amplitude, cumpre o seu papel no sentido de "estimular desde uma política nacional até a implantação de dispositivos que sejam provocadores de mudança no processo de trabalho cotidiano" (PINTO; SOUSA; FLORÊNCIO, 2013, P. 7).

CUNHA, M. S.; SÁ, M. C. Home visits within the Family Health Strategy (FHS): the challenges of moving into the territory. Interface: comunicação, saúde, educação, Botucatu, v. 17, n. 44, p. 61-73, jan./mar. 2013.

ESPOSTI, C. D. D. et al. O processo de trabalho do técnico em saúde bucal e suas relações com a equipe de saúde bucal na Região Metropolitana da Grande Vitória, Espírito Santo, Brasil. Saúde e Sociedade, São Paulo, v. 21, n. 2, p. 372-385, jun. 2012.

FACCIN, D.; SEBOLD, R.; CARCERERI, D. L. Processo de trabalho em saúde bucal: em busca de diferentes olhares para compreender e transformar a realidade. Ciência \& Saúde Coletiva, Rio de Janeiro, v. 15, n. 1, p. 1643-1652, jun. 2010.

\section{INSTITUTO BRASILEIRO DE GEOGRAFIA E} ESTATÍsTICA. Cidades. Censo Demográfico 2010. Disponível em: <ftp://ftp.ibge.gov.br/Censos/Censo_ Demografico_2010/Caracteristicas_Gerais_Religiao_ Deficiencia/caracteristicas_religiao_deficiencia.pdf />. Acesso em: 17 abr. 2015.

MATTOS, G. C. M. et al. A inclusão da equipe de saúde bucal na Saúde da Família: entraves, avanços e desafios. Ciência \& Saúde Coletiva, Rio de Janeiro, v. 19, n. 2, p. 373-382, fev. 2014.

MERHY, E. E. O ato de cuidar: a alma dos serviços de saúde. In: BRASIL. Ministério da Saúde. Secretaria de Gestão do Trabalho e da Educação na Saúde. Departamento de gestão da Educação na Saúde. VER SUS Brasil: cadernos de textos. Brasília, DF: Ministério da Saúde, 2004. 
Saúde: a cartografia do trabalho vivo em ato. 3 ed. São Paulo: Hucitec, 2007.

PEREIRA, C. R. S. et al. Impacto da Estratégia Saúde da Família sobre indicadores de saúde bucal: análise em municípios do Nordeste brasileiro com mais de 100 mil habitantes. Cad. Saude Publica, v. 28, n. 3, p. 449$462,2012$.

PINTO, H. A.; SOUSA, A.; FLORÊNCIO, A. R. O Programa Nacional de Melhoria do Acesso e da Qualidade da Atenção Básica: Reflexões sobre o seu desenho e processo de implantação. Revista Eletrônica de Comunicação, Informação \&t Inovação em Saúde, Rio de Janeiro, v.6, n. 2, ago. 2012. Disponível em: <http:// www.reciis.icict.fiocruz.br/index.php/reciis/article/ viewArticle/624/1083>. Acesso em: 10 abr. 2014.

REIS, M. A. S. et al. Labor process organization in a family health unit: challenges to practice change. Interface: comunicação, saúde, educação, Botucatu, v. 11, n. 2, p. 655-666, 2007

SANGLARD-OLIVEIRA, C. A. O. et al. Atribuições dos Técnicos em Saúde Bucal na Estratégia Saúde da Família em Minas Gerais, Brasil. Ciência \&t Saúde Coletiva, Rio de Janeiro, v. 18, n. 8, ago. 2013, p.

2453-2460.

SILVA, K. L. et al. Atenção domiciliar como mudança do modelo tecnoassistencial. Rev de Saúde Pública, São Paulo, v. 44, n. 1, p. 166-176, fev. 2010.

VELOSO, R. C.; ARAÚJO, M. R. N. Avaliação da resolutividade do programa saúde da família em municípios de pequeno porte no Estado de Minas Gerais. Revista de APS, v. 12, n. 3, p. 238-243, 2009.

\footnotetext{
Recebido para publicação em abril de 2014 Versão final em fevereiro de 2015

Conflito de interesse: inexistente

Suporte financeiro: não houve
} 